\title{
Competition between color salience and perceptual load during visual selection can be biased by top-down set
}

\author{
Adam T. BigGS ANd Bradley S. Gibson \\ University of Notre Dame, Notre Dame, Indiana
}

\begin{abstract}
Visual salience and perceptual load may both influence the efficiency of visual selection. Recently, Gibson and Bryant (2008) showed that perceptual load can dominate color salience in a distractor interference paradigm. The purpose of the present study was to investigate the possibility that knowledge (of color or load) may modulate the relative operation of these two mechanisms. Consistent with previous findings, perceptual load dominated color salience, but only in certain contexts in which display load was mixed and high-load displays preceded other high-load displays. More important, color salience dominated perceptual load in other contexts in which display load was mixed and low-load displays preceded high-load displays. In addition, color salience also dominated perceptual load in contexts in which display load was fixed and advance knowledge of load was available. Altogether, the present findings suggest that the competition between color salience and perceptual load can vary as a function of task context, thereby supporting top-down accounts, although the precise aspect of task context remains to be identified.
\end{abstract}

Attention researchers have long debated where in the stream of processing visual selection occurs. Over the past 15 years, Lavie and her colleagues (Lavie, 1995, 2005; Lavie \& Cox, 1997; Lavie \& Tsal, 1994) have suggested that the processing of both relevant and irrelevant items may proceed automatically, and in parallel, until attentional resources are consumed, at which point relevant items may be prioritized over irrelevant items. According to this perceptual load hypothesis, the selection of taskirrelevant items should appear to occur relatively late in the stream of processing, when the processing of taskrelevant items consumes relatively few resources, whereas the selection of task-irrelevant items should appear to occur relatively early in the stream of processing, when the processing of task-relevant items consumes relatively many resources (Lavie, 1995, 2005; Lavie \& Cox, 1997; Lavie \& Tsal, 1994).

Evidence in support of the perceptual load hypothesis has been obtained primarily from various distractor interference paradigms in which a target is presented with a distractor that is compatible, incompatible, or neutral with respect to the identity of the target item (see Lavie, 2005 , for a review). In addition, perceptual load is typically manipulated by varying the number of nontarget items that are presented along with the target and distractor items (but see Lavie, 1995, and Lavie \& Cox, 1997, for variations). In these studies, a late versus early locus of selection is associated with the presence or absence, respectively, of a distractor interference effect, which is typically defined as the difference in response time (RT) between the incompatible and neutral detractor conditions (Lavie, 1995). Previous studies have provided support for the perceptual load hypothesis by demonstrating the presence of a significant distractor interference effect under conditions of low perceptual load (signifying a relatively late locus of selection) and the absence of a significant distractor interference effect under conditions of high perceptual load (signifying a relatively early locus of selection; see Lavie, 2005, for a review).

However, other researchers have argued that this pattern of distractor interference may be more attributable to changes in distractor salience than to changes in perceptual load as the number of nontarget items increases. For instance, Eltiti, Wallace, and Fox (2005) argued that the onset of the distractor may be more salient, and therefore more likely to attract focal attention (Theeuwes, 1991, 1992, 1994, 2004), when only the target and distractor appear in the display (as in the low-load condition). In contrast, the distractor may be less salient, and therefore less likely to attract focal attention, when several additional items also onset simultaneously with the target and distractor in the display (as in the high-load condition). In addition to perceptual salience, Eltiti et al. also considered whether the attentional set of the observer might mediate the distractor's ability to attract focal attention and cause distraction (Burnham, 2007; Folk, Remington, \& Johnston, 1992; Gibson \& Kelsey, 1998). For instance, an onsetting distractor may be more likely to attract focal 
attention and cause interference than an offsetting distractor when the target is also defined by onset.

Eltiti et al. (2005, Experiment 3) investigated whether attentional set and perceptual salience may influence the occurrence of distractor interference by crossing the defining feature of the target (onset vs. offset) with the defining feature of the distractor (onset vs. offset) under conditions of low perceptual load to ensure that both defining features remained salient. If set and salience are important, distractor interference should occur only when the defining feature of the distractor is consistent with the defining feature of the target. In contrast, if perceptual load is important, distractor interference should occur regardless of the relation between the target and the distractor, because display load was always low. Their findings showed that an onsetting (but not an offsetting) distractor produced interference when the target was defined by onset; however, both an onsetting and an offsetting distractor produced interference when the target was defined by offset (see also Cosman \& Vecera, 2009).

The findings reported by Eltiti et al. (2005) are important, because they suggest that some perceptual features, such as abrupt onset, may cause distraction because of their relative salience (regardless of the current behavioral goals of the observer), whereas other perceptual features, such as offset, may cause distraction only because of their relevance to the current behavioral goals of the observer. According to this perspective, selection will appear to occur relatively late in the stream of processing, when the distractor is capable of attracting focal attention (either via its salience or via its relation to attentional set), whereas selection will appear to occur relatively early in the stream of processing, when the distractor is not capable of attracting focal attention. In other words, according to this perspective, the locus of selection appears to be determined by attentional capture, not perceptual load.

Recently, Gibson and Bryant (2008) also examined the relative influence of perceptual load and visual salience on the locus of selection using a distractor interference paradigm in which the distractor appeared as a salient color singleton (see Figure 1). In this task, observers searched for a target among a variable number of nontarget items that all appeared in a common color while also attempting to ignore a compatible or incompatible distractor that appeared as a color singleton. Previously, Theeuwes and
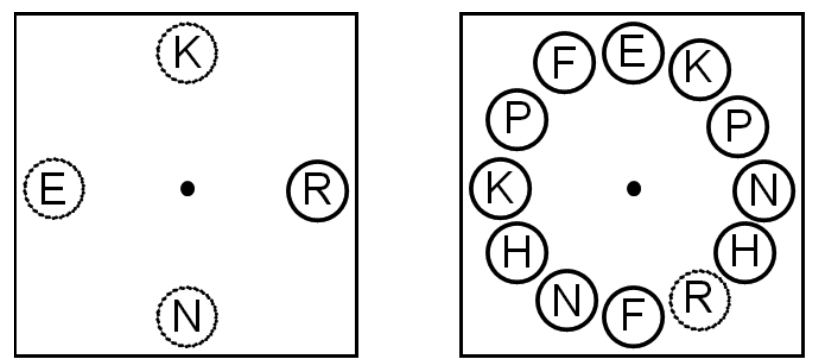

Figure 1. Search displays used in Experiments 1 and 2. Dotted circles indicate red and solid circles indicate green. Both displays depict incompatible singleton distractors.
Burger (1998) used this paradigm and concluded that salient color singletons can capture focal attention on the basis of the presence of a significant distractor interference effect. However, Theeuwes and Burger did not provide a strong manipulation of perceptual load in their study.

In contrast, Gibson and Bryant (2008) compared the magnitude of the distractor interference effect across display sizes of 4 and 12 items, and, consistent with the perceptual load hypothesis, they found significant distractor interference in the low-load (4-item) condition, but not in the high-load (12-item) condition. Note that the lack of distractor interference observed in the high-load condition cannot be attributed to a decrease in salience in that condition, because the color singleton should have become more salient as the number of homogenously colored items increased (cf. Eltiti et al., 2005). The findings reported by Gibson and Bryant therefore suggest that attentional capture by salient distractors cannot provide a complete account of the distractor interference effects that have been observed in the visual selection literature.

However, it should be noted that distractor interference was computed differently in the Gibson and Bryant (2008) study than in the Eltiti et al. (2005) study. More specifically, like many previous researchers who have investigated the perceptual load hypothesis (Lavie, 1995, 2005), Eltiti et al. measured distractor interference by subtracting performance in their neutral distractor condition from performance in their incompatible condition (I - N; they did not include a compatible distractor condition). In contrast, Gibson and Bryant measured distractor interference by subtracting performance in their compatible distractor condition from performance in their incompatible distractor condition (I - C; they did not include a neutral distractor condition; see also, Theeuwes \& Burger, 1998). This difference is potentially important, because compatible distractors may influence performance via both attentional and nonattentional mechanisms.

Moreover, the nonattentional mechanisms may have opposing effects on RTs. For instance, Lavie (1995) suggested that compatible distractors might shorten RTs relative to neutral distractors because of redundancy gains on signal activation, response selection, or both (Eriksen \& Eriksen, 1979; Miller, 1982; Santee \& Egeth, 1982); alternatively, compatible distractors might lengthen RTs relative to neutral distractors because of the inhibitory processing of target-specific features in low-level vision (Bjork \& Murray, 1977; Estes, 1972; Santee \& Egeth, 1982). Thus, given that Gibson and Bryant (2008) defined distractor interference in terms of $\mathrm{I}-\mathrm{C}$, the observed pattern of distractor interference may reflect the differential operation of nonattentional mechanisms in the compatible distractor condition rather than the operation of perceptual load.

This issue was addressed in the present experiments by combining Gibson and Bryant's (2008) distractor interference paradigm with Eltiti et al.'s (2005) measure of distractor interference. If distractor interference is determined by perceptual load, there should be significant distractor interference observed in the low-load condition, but not in the high-load condition. In contrast, if distractor interfer- 
ence is determined by attentional capture, the distractor interference effect observed in the high-load condition should be just as large as (or larger than) the distractor interference effect observed in the low-load condition.

In addition to providing a better measure of distractor interference, in the present study we also provided a more thorough investigation of the role of task set on the relative operation of attentional capture and perceptual load by manipulating two forms of knowledge. In order to ignore the irrelevant color distractor in this paradigm, we assumed that observers must first parse the display into relevant and irrelevant segments on the basis of color (see also Theeuwes \& Burger, 1998). The first manipulation that might influence task set therefore involved varying the observers' color knowledge of the target and distractor. More specifically, current evidence has distinguished between two feature-processing modes: singleton detection and feature search (Bacon \& Egeth, 1994; Lamy \& Egeth, 2003). In singleton detection, observers search for feature differences, whereas in feature search, they search for specific feature values. This distinction is important, because attentional capture by irrelevant singletons may be more likely when observers are engaged in singleton detection (Bacon \& Egeth, 1994). Moreover, these two search modes may vary depending on the observers' feature knowledge. For instance, observers can only engage in singleton detection when the specific feature values associated with the singleton and nonsingletons are unknown, whereas the observers may (at least in principal) engage in feature search when the specific feature values are known in advance.

The distinction between singleton detection and feature search may be important in the present study, because the observers in Gibson and Bryant's (2008) critical Experiment 2 were provided specific color knowledge about the target and nontargets (but not about the distractor), which may have been sufficient to promote feature search. Thus, the procedure may not have been optimal for observing attentional capture. In order to address this issue, color knowledge was manipulated by providing participants either full knowledge or no knowledge of the color of the target and distractor. In the full-knowledge-of-color condition, the colors of the target, nontargets, and distractor remained constant throughout the entire experiment, whereas in the no-knowledge-of-color condition, the colors of the target, nontargets, and distractor changed randomly from trial to trial (with the standard constraint that the target and nontargets always appeared the same color). If the relative influence of attentional capture versus perceptual load on visual selection can vary as a function of color knowledge, a larger distractor interference effect may be observed under conditions of high perceptual load in the no-knowledge-of-color condition than in the fullknowledge-of-color condition.

The second manipulation that might influence task set involved varying observers' knowledge of display load. High- and low-load displays are routinely intermixed in studies of attentional capture, whereas high- and low-load displays are routinely blocked in studies of perceptual load (see, e.g., Lavie, 1995). Thus, observers typically lack knowledge of load in studies of attentional capture but not in studies of perceptual load.

Theeuwes, Kramer, and Belopolsky (2004) examined this issue by randomly mixing or blocking high- and lowload displays using a paradigm in which perceptual load was manipulated via the perceptual similarity of the target and nontargets (Lavie \& Cox, 1997; note that Theeuwes et al. used I - C as their measure of distractor interference). Theeuwes et al. found that distractor interference was modulated by intertrial contingencies that arose from the random mixture of high- and low-load displays, in that observers were more likely to attend to the irrelevant distractor in the high-load condition when the preceding trial was low in perceptual load than when it was high in perceptual load (see also Fournier \& Shorter, 2001, for other evidence that distractor interference may be modulated by knowledge-of-target-probabilities under conditions of high perceptual load). This finding in turn suggested that the effects of perceptual load may be modulated by the attention-allocation set of the observer. In particular, Theeuwes et al. suggested that attention was more likely to be divided across a current high-load display when the preceding display was low-load than when the preceding display was high-load, which in turn made the observers more vulnerable to the distractor. In contrast, Theeuwes et al. observed consistent distractor interference in the low-load condition, regardless of the display load of the preceding trial, suggesting that the distractor interference observed in the low-load condition was not modulated by the attention-allocation set of the observer. In addition, Theeuwes et al. also observed the typical load $\times$ distractor interference interaction predicted by the perceptual load account in the blocked condition.

The intertrial contingencies that occur when observers have no knowledge of load may be important in the present context; Belopolsky, Zwaan, Theeuwes, and Kramer (2007) recently argued that capture by salient singletons may depend on the width of the attentional window (see also Gibson \& Peterson, 2001; Theeuwes, 2004). This is because attentional capture depends on the computation of visual salience, and the computation of visual salience in turn requires displaywide comparisons, which may only be possible when attention is distributed across the display. Consequently, attentional capture may be more likely to occur when attention is initially allocated more widely across the display, rather than more narrowly to individual items. If the relative influence of attentional capture versus perceptual load on visual selection can vary as a function of knowledge of load in the present paradigm, the magnitude of the distractor interference effect observed under conditions of high perceptual load may vary across the noknowledge-of-load condition (where intertrial contingencies may occur) and the full-knowledge-of-load condition (where intertrial contingencies do not occur).

Note that Gibson and Bryant (2008) also randomly mixed high- and low-load displays (as did Theeuwes \& Burger, 1998); however, contrary to Theeuwes et al. (2004), Gibson and Bryant did not observe any significant intertrial contingencies in the high-load condition of their Experiment 2, although they did observe a trend 
in the predicted direction. Moreover, it should be noted that sample size was relatively small $(N=10)$ in Gibson and Bryant's experiment. Given the potential importance of knowledge-of-load manipulations for understanding top-down effects on visual selection, in the present study we re-examined this issue by investigating whether significant intertrial contingencies could be observed in this paradigm under conditions of random mixing (Experiment 1) versus blocking (Experiment 2) when much larger samples $(n>50)$ were used in each knowledge-of-load condition and a more appropriate measure of distractor interference $(\mathrm{I}-\mathrm{N})$ was used.

In summary, the results reported by Eltiti et al. (2005) and Gibson and Bryant (2008) suggest that either or both of two bottom-up mechanisms - attentional capture and perceptual load - can influence the efficiency of visual selection in any given situation. However, questions remain regarding the measurement of distractor interference in Gibson and Bryant's study, as well as the possible role that knowledge (of color or load) may play on the relative operation of these two mechanisms. Accordingly, the purpose of the present study was to further investigate the measurement and boundary conditions associated with the operation of these two mechanisms within the context of Theeuwes and Burger's (1998) color singleton paradigm.

\section{EXPERIMENT 1}

Gibson and Bryant (2008) measured the effect of color salience on the efficiency of visual selection under conditions of high and low load and found evidence that perceptual load dominated salience. In Experiment 1, we investigated whether group differences in the knowledge of color could increase the effect of color salience on visual selection using an unbiased measure of distractor interference $(\mathrm{I}-\mathrm{N})$. If so, greater distractor interference should be observed in the no-knowledge-of-color condition than in the full-knowledge-of-color condition when display load is high. In addition, the possible role of color salience was thought to be further increased in this experiment by randomly mixing display load, thereby creating a no-knowledge-of-load condition in which observers' attention-allocation set for an upcoming display may be influenced by the load of the current display (the contrasting full-knowledge-of-load condition will be examined in Experiment 2).

\section{Method \\ Participants. Fifty-eight undergraduate students from the Uni- versity of Notre Dame participated in the experiment for partial completion of a course requirement. Twenty-nine undergraduates were randomly assigned to the full-knowledge-of-color condition, and 29 were assigned to the no-knowledge-of-color condition. The results from 2 individuals ( 1 from each knowledge condition) were discarded because of error rates greater than $20 \%$. All of the observ- ers reported normal or corrected-to-normal visual acuity without color deficits.}

Stimuli and Apparatus. The search displays were similar to those used by Gibson and Bryant (2008) and are shown in Figure 1. Four or 12 items were placed at equal intervals on an imaginary circle with radius of $3.2^{\circ}$ visual angle at a viewing distance of $57 \mathrm{~cm}$.
Each item was a letter subtending $0.76^{\circ}$ in height and $0.55^{\circ}$ in width surrounded by a ring with a diameter of $1.4^{\circ}$. The rings were either green $\left(18.81 \mathrm{~cd} / \mathrm{m}^{2}\right)$ or red $\left(18.81 \mathrm{~cd} / \mathrm{m}^{2}\right)$, but the letters were always gray $\left(18.48 \mathrm{~cd} / \mathrm{m}^{2}\right)$. Note that the center-to-center distance between items was smaller in the 12 -item display $\left(1.66^{\circ}\right)$ than it was in the 4-item display $\left(4.53^{\circ}\right)$, which may be critical for creating high- and low-load conditions. For instance, Torralbo and Beck (2008) recently argued that high- and low-load conditions may reflect differences in competitive spatial interactions that are dependent on the relative density of display stimuli, with more tightly packed stimuli inducing greater competition and higher perceptual load. Responses were measured with a custom-made button box (Lafayette Instruments) and recorded to the nearest millisecond. Timing and presentation of stimuli were controlled by the DMDX experimental software program (Forster \& Forster, 2003).

Procedure. A fixation dot appeared for $500 \mathrm{msec}$, followed by the search display. The search display was equally likely to contain 4 (low load) or 12 (high load) items. Each search display contained a variable number of commonly colored items (either 3 or 11) and a uniquely colored item. The commonly colored items always contained one of the two target letters (E or R) and a variable number of nontarget letters $(\mathrm{H}, \mathrm{P}, \mathrm{N}, \mathrm{K}$, or F). Two of the nontarget letters were randomly selected from this set without replacement in the 4-item condition, and all five of the nontargets were selected twice in the 12 -item condition. The uniquely colored item contained an incompatible letter ( $\mathrm{R}$ if the target was $\mathrm{E}$ or $\mathrm{E}$ if the target was $\mathrm{R}$ ), a neutral letter $(T)$, or a compatible letter ( $R$ if the target was $R$ or $E$ if the target was E) with equal probability. The participants pressed one of two buttons to indicate which target letter they saw in the display.

The observers were instructed to keep their eyes fixated on the dot and to search for the target among the rings while ignoring the item inside the uniquely colored ring. The target displays remained visible until a response was made (or until $4 \mathrm{sec}$ had elapsed). Prior knowledge of the color of the target, nontarget, and distractor was included as a between-subjects manipulation. The colors of the target, nontargets, and distractor rings were fixed in the fullknowledge-of-color condition. Half of the observers in the fullknowledge-of-color condition saw red targets and nontargets and a green distractor, whereas, the other half of the observers saw the opposite color assignment. The colors of the target, nontargets, and distractor rings were random in the no-knowledge-of-color condition, such that the participants could see red targets and nontargets with a green distractor or green targets and nontargets with a red distractor on any given trial; however, target and nontarget rings were always the same color. Each observer performed a total of 864 experimental trials with a representative block of 32 practice trials presented beforehand, containing an equal number of the possible combinations (low-load incompatible distractor; low-load neutral distractor, etc.). A different random order of experimental trials was presented to each observer.

\section{Results and Discussion}

Mean correct RTs are depicted in Figure 2 as a function of perceptual load and distractor compatibility in each of the two knowledge-of-color conditions. The corresponding error rates are listed in Table 1. In this and all subsequent experiments reported in this article, incorrect key responses and response latencies greater than $4,000 \mathrm{msec}$ or less than $200 \mathrm{msec}$ were treated as errors and excluded from the RT analyses (note that less than $1 \%$ of the data were excluded on the basis of the latency criteria in each of the three experiments). A three-way mixed ANOVA was conducted on mean correct RTs and error rates with perceptual load (low vs. high) and distractor compatibility (incompatible vs. neutral vs. compatible) as the two within-subjects variables and knowledge-of-color (full vs. 


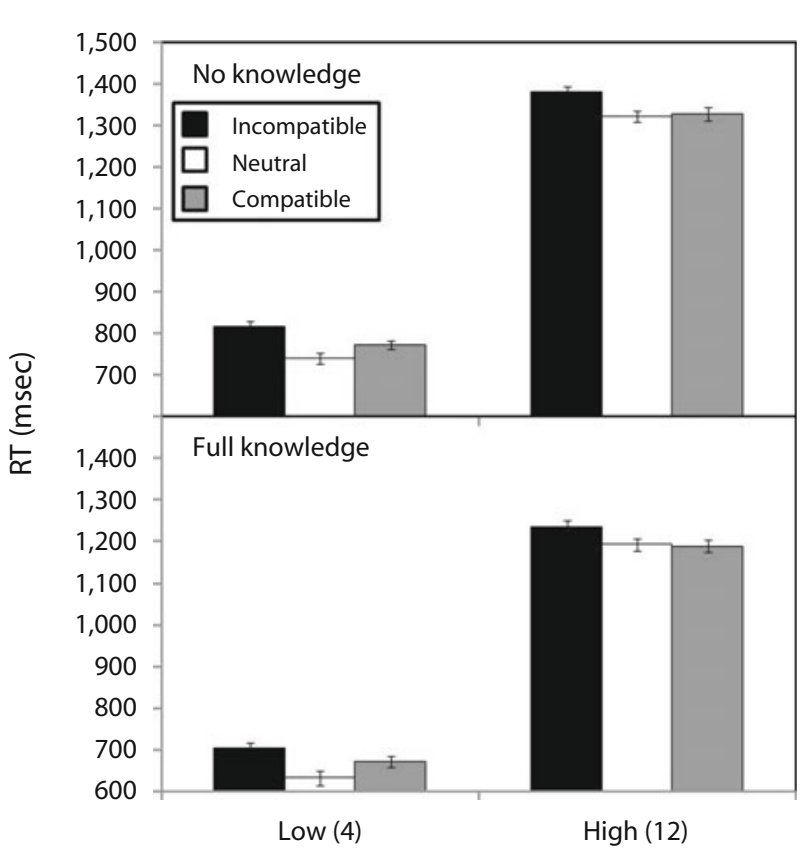

Perceptual Load

Figure 2. Mean correct response times (RTs) as a function of perceptual load and distractor compatibility in the no-knowledgeof-color (top panel) and full-knowledge-of-color (bottom panel) conditions in Experiment 1. The observers in Experiment 1 had no advance knowledge of perceptual load. Error bars represent the standard error of the mean with between-subjects variation removed (Loftus \& Masson, 1994).

no) as the sole between-subjects variable. All interpretations of the data were identical using both the sphericity assumption and the Greenhouse-Geisser method.

With respect to mean correct RTs, there was a significant main effect of perceptual load $[F(1,54)=1,022.38$, $\left.p<.001, \eta_{\mathrm{p}}^{2}=.95\right]$, indicating shorter RTs in the lowload condition than in the high-load condition. There was also a significant main effect of distractor compatibility $\left[F(2,108)=45.31, p<.001, \eta_{\mathrm{p}}^{2}=.46\right]$, indicating shorter RTs in the neutral $(M=972 \mathrm{msec})$ and compatible $(M=$ $990 \mathrm{msec}$ ) conditions than in the incompatible condition $(M=1,033 \mathrm{msec})$. In addition, there was a signifi- cant main effect of knowledge of color $[F(1,54)=6.89$, $\left.p<.025, \eta_{\mathrm{p}}^{2}=.11\right]$, indicating shorter RTs in the fullknowledge-of-color condition $(M=937 \mathrm{msec})$ than in the no-knowledge-of-color condition $(M=1,060 \mathrm{msec})$. This latter finding is important because it suggests that the observers used their color knowledge to parse the search display into relevant and irrelevant regions.

More important, there was a significant two-way interaction between perceptual load and distractor compatibility $\left[F(2,108)=4.38, p<.025, \eta_{\mathrm{p}}^{2}=.08\right]$. Consistent with the perceptual load hypothesis, the distractor interference effect $(\mathrm{I}-\mathrm{N})$ was significantly larger in the low-load condition $(M=74 \mathrm{msec})$ than in the high-load condition $(M=49 \mathrm{msec})\left[F(1,54)=6.28, p<.025, \eta_{\mathrm{p}}^{2}=.10\right]$. However, despite this significant reduction, the distractor interference effect was found to be significant in both the low-load and high-load conditions $[t(55)=11.14, p<$ .001 and $t(55)=5.60, p<.001$, respectively].

The significant, albeit reduced, effect of distractor interference observed in the high-load condition therefore suggests a possible role for attentional capture in determining the efficiency of visual selection. One way to substantiate the conclusion that attentional capture may play a role in the distractor interference effect is to examine whether the magnitude of this effect varies as a function of color knowledge. In particular, attentional capture has been argued to depend on the use of a singleton detection processing strategy, which may in turn be more likely in the no-knowledge-of-color condition than in the fullknowledge-of-color condition (Bacon \& Egeth, 1994). If this contingency is correct, the distractor interference effect should be larger in the no-knowledge-of-color condition than in the full-knowledge-of-color condition. However, as can be seen in Figure 2, the distractor interference effect was very similar across the two knowledge-of-color conditions. As a result, none of the interactions involving knowledge of color approached significance in the present study (all $F \mathrm{~s}<1$ ).

Note that Belopolsky et al. (2007) have recently argued that capture by salient singletons may depend not directly on feature knowledge per se, but on the width of the attentional window. This is because attentional capture depends on the computation of visual salience, and the computation of visual salience in turn requires displaywide

Table 1

Mean Error Rates (in Percentages) As a Function

of Perceptual Load and Compatibility in Each of the Two Knowledge-of-Color Conditions in Experiment 1

\begin{tabular}{|c|c|c|c|c|c|c|c|c|}
\hline \multirow{3}{*}{$\begin{array}{l}\text { Perceptual } \\
\text { Load }\end{array}$} & \multicolumn{6}{|c|}{ Distractor Compatibility } & & \\
\hline & \multicolumn{2}{|c|}{ Incompatible (I) } & \multicolumn{2}{|c|}{ Neutral $(\mathrm{N})$} & \multicolumn{2}{|c|}{ Compatible (C) } & \multicolumn{2}{|c|}{ Interference } \\
\hline & $M$ & $S E$ & $M$ & $S E$ & $M$ & $S E$ & $\mathrm{I}-\mathrm{N}$ & $\mathrm{C}-\mathrm{N}$ \\
\hline \multicolumn{9}{|c|}{ No Knowledge } \\
\hline Low & 6.16 & 0.49 & 3.36 & 0.31 & 4.20 & 0.41 & 2.80 & 0.84 \\
\hline High & 7.14 & 0.49 & 5.45 & 0.33 & 6.15 & 0.37 & 1.69 & 0.70 \\
\hline \multicolumn{9}{|c|}{ Full Knowledge } \\
\hline Low & 4.79 & 0.48 & 3.10 & 0.33 & 3.13 & 0.34 & 1.69 & 0.03 \\
\hline High & 7.31 & 0.43 & 5.93 & 0.44 & 6.21 & 0.23 & 1.38 & 0.28 \\
\hline
\end{tabular}

Note-The observers in Experiment 1 had no advance knowledge of perceptual load. Between-subjects variation was removed from $S E$ s. 
comparisons, which may only be possible when attention is distributed across the display. Consequently, attentional capture may be more likely to occur when attention is initially allocated more widely across the display than when it is allocated more narrowly to individual items. We investigated this possibility in the present study by investigating intertrial contingencies that result from the random intermixing of high- and low-load trials (Theeuwes et al., 2004). In the present study, we hypothesized that observers might allocate their attention more widely when load is low, whereas they might allocate their attention more narrowly when load is high. If so, the allocation strategy used on trial $n-1$ might influence attentional capture by singletons on trial $n$.

A four-way mixed ANOVA was conducted on mean correct RTs, with perceptual load on trial $n$ (low vs. high), perceptual load on trial $n-1$ (low vs. high), and distractor compatibility (incompatible vs. neutral) as the three within-subjects variables and knowledge of color (full vs. no) as the sole between-subjects variable. As was expected, there was a significant three-way interaction among perceptual load on trial $n$, perceptual load on trial $n-1$, and compatibility $[F(1,54)=5.71, p<.025$, $\left.\eta_{\mathrm{p}}^{2}=.10\right]$. In addition, although the main effect of knowledge of color was significant $[F(1,54)=7.26, p<.01$, $\left.\eta_{\mathrm{p}}^{2}=.12\right]$, this variable did not interact with any of the other variables (all $p \mathrm{~s}>.10$ ). The results of this intertrial contingency analysis are listed in Table 2, averaged over the two knowledge-of-color conditions. Consistent with the attention-allocation account, a significant distractor interference effect $(67 \mathrm{msec})$ was found in the highload condition when the preceding trial was of low load $[t(55)=5.62, p<.001]$, but a nonsignificant distractor interference effect $(21 \mathrm{msec})$ was found when the preceding trial was of high load $[t(55)=1.40, p>.10]$. These findings suggest that a relatively wide distribution of attention may be necessary to observe distractor interference in the high-load condition, perhaps because a wide distribution of attention is necessary for the detection of salience (Belopolsky et al., 2007; Gibson \& Peterson, 2001; Theeuwes, 2004). In contrast, significant distractor interference effects (both $73 \mathrm{msec}$ ) were found in the two low-load conditions, regardless of whether the preceding trial was of high or low load (both $p$ s $<.001$ ). These find- ings suggest that a relatively wide distribution of attention may not be necessary to observe distractor interference in the low-load condition, perhaps because distractor interference can be determined by either salience or low load in this condition. ${ }^{1}$

For the sake of completeness, we also compared mean correct RTs in the compatible and neutral distractor conditions. Surprisingly, RTs were generally longer in the compatible distractor condition $(M=990 \mathrm{msec})$ than in the neutral distractor condition $(M=972 \mathrm{msec})$, perhaps reflecting inhibition of target-specific features (Bjork \& Murray, 1977; Estes, 1972; Santee \& Egeth, 1982). In addition, the RT difference between the compatible and neutral distractor conditions was significant when load was low $[t(55)=6.68, p<.001]$, but not when load was high $[t(55)=0.10, p>.90]$. These results were unexpected from the standpoint of attentional selection and suggest that other, nonattentional mechanisms influence RTs in the compatible distractor condition. Note that because compatible RTs were longer than expected in the lowload condition, the use of I - C (as opposed to I - N) in the present context would have tended to underestimate the magnitude of distractor interference observed in the low-load condition but not in the high-load condition. Consequently, the use of I - C (as opposed to I - N) would have biased interpretation toward attentional capture rather than perceptual load. Thus, the present findings do not challenge Gibson and Bryant's (2008) conclusion that perceptual load can dominate attentional capture; indeed, their experiment may have been biased against this conclusion.

Nevertheless, these findings serve as a methodological warning against using $\mathrm{I}-\mathrm{C}$ to estimate the magnitude of distractor interference. Perhaps for this reason, it has become commonplace to include only the neutral and incompatible distractor conditions in studies of perceptual load (see, e.g., Eltiti et al., 2005; Lavie, 2005). However, although we agree that the compatible condition should not be used in the calculation of distractor interference, we do not endorse excluding this condition from the experimental procedure altogether, because doing so may enable strategies that cloud interpretation of distractor effects. For instance, using the present paradigm, in which only the neutral and incompatible conditions were included, we

Table 2

Mean Correct Response Times (in Milliseconds)

As a Function of Intertrial Contingencies in Each of the Two Perceptual Load Conditions in Experiment 1

\begin{tabular}{|c|c|c|c|c|c|}
\hline \multirow[b]{3}{*}{ Intertrial Contingency } & \multicolumn{4}{|c|}{ Distractor Compatibility } & \multirow{3}{*}{$\begin{array}{c}\text { Interference } \\
(\mathrm{I}-\mathrm{N})\end{array}$} \\
\hline & \multicolumn{2}{|c|}{ Incompatible } & \multicolumn{2}{|c|}{ Neutral } & \\
\hline & $M$ & $S E$ & $M$ & $S E$ & \\
\hline \multicolumn{6}{|c|}{ Low Load } \\
\hline Same (from low to low) & 759 & 10.0 & 686 & 10.6 & $73^{*}$ \\
\hline Switch (from high to low) & 760 & 10.2 & 687 & 11.4 & $73^{*}$ \\
\hline \multicolumn{6}{|c|}{ High Load } \\
\hline Same (from high to high) & 1,303 & 12.8 & 1,282 & 14.7 & 21 \\
\hline Switch (from low to high) & 1,308 & 13.5 & 1,241 & 10.9 & $67^{*}$ \\
\hline
\end{tabular}

${ }^{*} p<.001$. 
have actually observed a significant -116 -msec distractor facilitation effect $(\mathrm{I}-\mathrm{N})$ under conditions of high perceptual load $[t(15)=2.92, p<.025]$ in an independent sample of 16 observers. One explanation for this unexpected distractor facilitation effect concerns the possibility that the observers in the high-load condition intentionally attended to the salient color distractor because its identity could be used to predict the identity of the target in the incompatible condition but not in the neutral condition. Thus, proper interpretation of the distractor interference effects vis-à-vis the perceptual load account requires the inclusion of all three compatibility conditions, although only the incompatible and neutral conditions should be used to estimate distractor interference.

An identical ANOVA was performed on error rates. The results indicated a significant main effect of perceptual load $\left[F(1,54)=42.30, p<.001, \eta_{\mathrm{p}}^{2}=.44\right]$ with more errors made in the high-load condition $(M=6.37 \%)$ than in the low-load condition $(M=4.12 \%)$, and a significant main effect of compatibility $[F(2,108)=23.16, p<.001$, $\left.\eta_{\mathrm{p}}^{2}=.30\right]$, with more errors observed in the incompatible condition $(M=6.35 \%)$ than in either the neutral $(M=$ $4.46 \%)$ or compatible $(M=4.92 \%)$ conditions. There was no significant main effect of knowledge of color $(F<1)$, and no significant interactions were observed in the error rate analysis. Thus, the RT findings do not appear to be compromised by a speed-accuracy trade-off.

\section{EXPERIMENT 2}

The results from Experiment 1 indicated that the presence of distractor interference in the high-load condition was modulated by intertrial contingencies that arose from the mixture of high- and low-load displays. This modulation, in turn, was interpreted to suggest that the color singleton did not capture attention in a purely bottomup fashion but, rather, was contingent on the attentionallocation set of the observer (see also Theeuwes et al., 2004). In particular, color salience may dominate visual selection when attention is widely distributed, whereas perceptual load may dominate visual selection when attention is more narrowly focused.

Note that other intertrial contingency effects involving trial-to-trial sequences of same or different target shapes have recently been reported by researchers using the additional singleton paradigm, and these effects have been interpreted to reflect bottom-up priming rather than topdown set (Pinto, Olivers, \& Theeuwes, 2005; but see Lamy \& Yashar, 2008, for contrary evidence that these effects reflect top-down set rather than bottom-up priming). Thus, it is important to consider whether the intertrial contingency effects involving trial-to-trial sequences of same or different display load that were observed in Experiment 1 might also reflect modulation by bottom-up priming rather than top-down set.

One minimal empirical requirement of the bottom-up priming account is that identical results should be obtained under identical trial-to-trial priming conditions, regardless of observers' knowledge of these priming conditions. Recall that the effect of distractor interference observed in the high-load condition of Experiment 1 was found to be relatively small $(M=21 \mathrm{msec})$ and nonsignificant when the load on trial $n-1$ was also high, whereas the effect of distractor interference observed in the low-load condition of Experiment 1 was found to be relatively large $(M=$ $73 \mathrm{msec}$ ) and significant when the load on trial $n-1$ was also low (although it should be noted that a similar result was also obtained in the low-load condition when the load on trial $n-1$ was high). Recall also that observers had no advance knowledge of display load in Experiment 1. If these intertrial contingency effects reflect bottom-up priming, a similar pattern of results should be obtained when the trial-to-trial load is fixed within a block and advance knowledge of display load is made available. That is, a significant perceptual load $\times$ distractor compatibility interaction should be observed, in which the effect of distractor interference is relatively small and nonsignificant in the high-load condition but relatively large and significant in the low-load condition.

Note that although such a result is required by the bottom-up priming account, it could also be consistent with the top-down set account. For instance, observers may only distribute their attention widely during blocks of low-load trials, and they may only distribute their attention narrowly during blocks of high-load trials. Consequently, other experiments will be required to distinguish the two accounts if the perceptual load $\times$ distractor compatibility interaction that is predicted by the bottom-up priming account is obtained. However, the top-down set account need not be bound by this empirical prediction; other empirical outcomes can be accommodated by this account because changes in performance can be linked to changes in knowledge or task context, even if these changes in performance are unexpected. Thus, the present experiment has the potential to rule out the bottomup priming account if different results are obtained for the full-knowledge-of-load (Experiment 2) and the noknowledge-of-load (Experiment 1) conditions.

\section{Method}

Participants. Fifty-six undergraduate students from the University of Notre Dame participated in the experiment for partial completion of a course requirement. Although knowledge of color did not have an effect in Experiment 1, we included this manipulation once again in Experiment 2 to mirror the design of Experiment 1 . Accordingly, 28 undergraduates were randomly assigned to the full-knowledge-of-color condition, and 28 were assigned to the no-knowledge-of-color condition. Results from 2 individuals (both in the no-knowledge-of-color condition) were discarded because of error rates greater than $20 \%$. All of the observers reported normal or corrected-to-normal visual acuity without color deficits.

Stimuli and Apparatus. The stimuli and apparatus were identical to those used in Experiment 1.

Procedure. The procedure was identical to that in Experiment 1, except that the high-load and low-load conditions were presented in two separate blocks. Experimental blocks were preceded by a practice session of 16 trials representing the load condition, and the order of block presentation was counterbalanced between observers.

\section{Results and Discussion}

Mean correct RTs are depicted in Figure 3 as a function of perceptual load and distractor compatibility in each of 
the two knowledge-of-color conditions. The corresponding error rates are listed in Table 3. A three-way mixed ANOVA was conducted on mean correct RTs and error rates with perceptual load (low vs. high) and distractor compatibility (incompatible vs. neutral) as the two withinsubjects variables and knowledge of color (full vs. no) as the sole between-subjects variable. Note that although compatible distractors were included in the experimental trials for methodological reasons, they were discarded from the analysis of distractor interference on the basis of the findings obtained in Experiment 1, which suggested that RTs in this condition might be influenced by nonattentional mechanisms.

With respect to mean correct RTs, there was a significant main effect of perceptual load $[F(1,52)=673.49$, $p<.001, \eta_{\mathrm{p}}^{2}=.93$ ], indicating shorter RTs in the lowload condition than in the high-load condition. There was also a significant main effect of distractor compatibility $\left[F(1,52)=288.49, p<.001, \eta_{\mathrm{p}}^{2}=.85\right]$, indicating shorter RTs in the neutral condition than in the incompatible condition. Unlike in Experiment 1, the main effect of knowledge of color did not approach significance $(F<1)$. More important, contrary to the bottom-up priming account, the magnitude of the distractor interference effect observed in the low-load condition $(M=77 \mathrm{msec})$ was very similar to the magnitude of the distractor interference effect observed in the high-load condition $(M=79 \mathrm{msec})$, leading to a nonsignificant perceptual load $\times$ distractor compat-

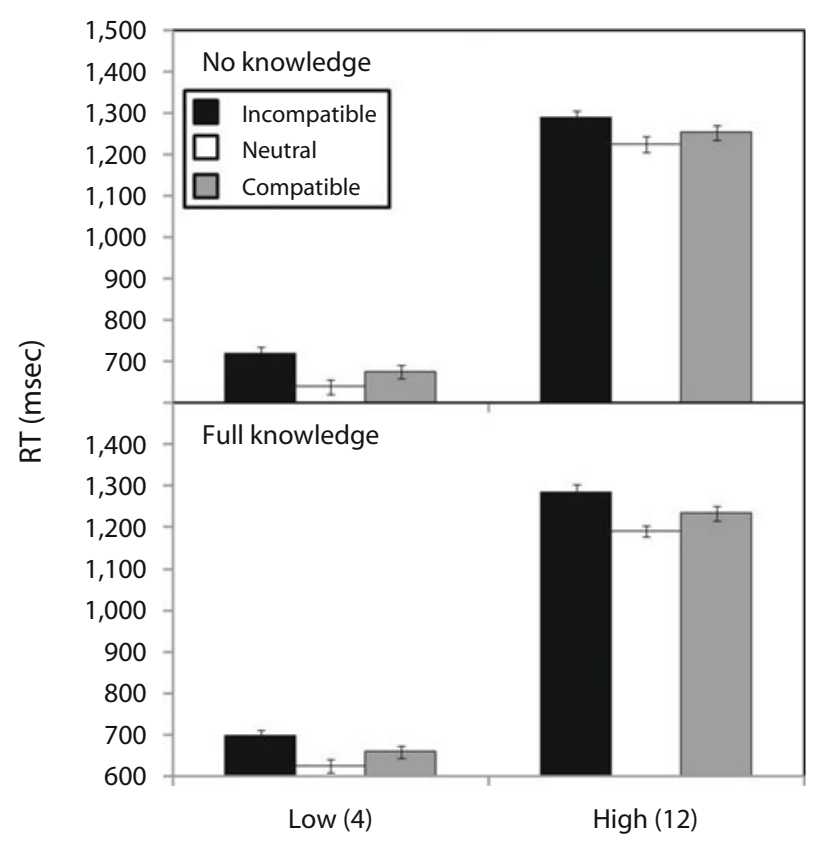

Perceptual Load

Figure 3. Mean correct response times (RTs) as a function of perceptual load and distractor compatibility in the no-knowledgeof-color (top panel) and full-knowledge-of-color (bottom panel) conditions in Experiment 1. The observers in Experiment 1 had full advance knowledge of perceptual load. Error bars represent the standard error of the mean with between-subjects variation removed (Loftus \& Masson, 1994). ibility interaction $(F<1)$. None of the other interactions was significant (all $p \mathrm{~s}>.05$ ).

Moreover, the effect of distractor interference observed in the high-load condition of the present experiment was significantly larger than the effect of distractor interference observed in the high-load condition of Experiment 1 when the load on trial $n-1$ was also high $[t(108)=$ $-3.37, p<.001]$. In fact, the effect of distractor interference observed in the high-load condition of the present experiment was actually more similar to the relatively large and significant $(M=67 \mathrm{msec})$ effect of distractor interference that was observed in the high-load condition of Experiment 1 when the load on trial $n-1$ was low $[t(108)=-0.85, p>.35]$. Likewise, the effect of distractor interference observed in the low-load condition of the present experiment was also very similar to the effect of distractor interference observed in the low-load condition of Experiment 1 when the load on trial $n-1$ was also low $[t(108)=-0.45, p>.65]$.

An identical ANOVA was performed on error rates. The results indicated a significant main effect of perceptual load $\left[F(1,52)=19.83, p<.001, \eta_{\mathrm{p}}^{2}=.28\right]$, with more errors made in the high-load condition $(M=6.49 \%)$ than in the low-load condition $(M=4.44 \%)$, and a significant main effect of distractor compatibility $[F(1,52)=23.16$, $\left.p<.001, \eta_{\mathrm{p}}^{2}=.30\right]$, with more errors observed in the incompatible condition $(M=6.58 \%)$ than in the neutral condition $(M=4.36 \%)$. There was no significant main effect of knowledge of color $(F<1)$.

Contrary to the RT results, a significant perceptual load $\times$ distractor compatibility interaction was observed $\left[F(1,52)=9.21, p<.01, \eta_{\mathrm{p}}^{2}=.15\right]$, indicating that the overall distractor interference effect was larger in the lowload condition $(M=3.12 \%)$ than in the high-load condition $(M=1.32 \%)$. This finding provides some evidence that distractor interference was modulated by perceptual load in the present experiment, although it should be noted that the effect of distractor interference was significant in both the high- and low-load conditions $[t(53)=2.95, p<$ .01 and $t(53)=6.41, p<.001$, respectively]. Thus, even these results are inconsistent with the bottom-up priming account. Moreover, although the three-way interaction between knowledge of color, perceptual load, and distractor compatibility was only marginally significant $[F(1,52)=$ $\left.2.83, p=.098, \eta_{\mathrm{p}}^{2}=.05\right]$, closer examination of the data revealed an unusually large effect of distractor interference in the no-knowledge-of-color condition relative to the full-knowledge-of-color condition when perceptual load was low ( $M=4.36 \%$ vs. $M=1.86 \%$, respectively) $[t(52)=2.77, p<.01]$. In contrast, the effect of distractor interference was nearly equal across the no- and fullknowledge-of-color conditions when perceptual load was high ( $M=1.58 \%$ vs. $M=1.07 \%$, respectively) $[t(52)=$ $.58, p>.55]$. This unexpected trend also contributed to the significant perceptual load $\times$ distractor compatibility interaction.

In summary, the bottom-up priming account of the intertrial contingency effects observed in Experiment 1 predicted a perceptual load $\times$ distractor compatibility interaction in Experiment 2, in which the effect of distractor 
Table 3

Mean Error Rates (in Percentages) As a Function of Perceptual Load and Compatibility in Each of the Two Knowledge-of-Color Conditions in Experiment 2

\begin{tabular}{|c|c|c|c|c|c|c|c|c|}
\hline \multirow[b]{3}{*}{ Perceptual Load } & \multicolumn{6}{|c|}{ Distractor Compatibility } & & \\
\hline & \multicolumn{2}{|c|}{ Incompatible (I) } & \multicolumn{2}{|c|}{ Neutral $(\mathrm{N})$} & \multicolumn{2}{|c|}{ Compatible (C) } & \multicolumn{2}{|c|}{ Interference } \\
\hline & $M$ & $S E$ & $M$ & $S E$ & $M$ & $S E$ & $\mathrm{I}-\mathrm{N}$ & $\mathrm{C}-\mathrm{N}$ \\
\hline \multicolumn{9}{|c|}{ No Knowledge } \\
\hline Low & 7.08 & 0.49 & 2.72 & 0.47 & 2.46 & 0.47 & 4.36 & -0.26 \\
\hline High load & 7.07 & 0.50 & 5.49 & 0.40 & 5.62 & 0.42 & 1.58 & 0.13 \\
\hline \multicolumn{9}{|c|}{ Full Knowledge } \\
\hline Low load & 4.92 & 0.55 & 3.05 & 0.39 & 2.75 & 0.48 & 1.87 & -0.30 \\
\hline High load & 7.24 & 0.47 & 6.18 & 0.46 & 6.48 & 0.58 & 1.06 & 0.30 \\
\hline
\end{tabular}

Note-The observers in Experiment 2 had full advance knowledge of perceptual load. Between-subjects variability was removed from $S E \mathrm{~s}$.

interference was significant under conditions of low load but not under conditions of high load. However, the results of Experiment 2 were inconsistent with this prediction: Surprisingly, providing advance knowledge of load actually increased the effect of distractor interference found in the high-load condition of the present experiment relative to providing no knowledge of load in Experiment 1, even when the intertrial load sequences were equated across the two experiments. Thus, on the basis of these findings, it seems reasonable to conclude that the relative effects of perceptual load and color salience on visual selection are contingent on differences in top-down set deriving from differences in task knowledge rather than from bottom-up priming. However, because the results deviated from those expected on the basis of the intertrial contingencies observed in Experiment 1, it appears that the present change in context also had a more profound impact on top-down set than originally expected. This issue will be discussed in greater detail in the General Discussion section.

Although the findings obtained in Experiment 2 provided support for the top-down set account, this conclusion may seem counterintuitive, given that these findings showed that color salience can dominate visual selection when the trial-to-trial load is fixed. Indeed, such findings could be interpreted to support a bottom-up capture account if considered in isolation (Theeuwes \& Burger, 1998). However, such findings cannot easily be interpreted to reflect bottom-up capture when they are interpreted in conjunction with the findings obtained in Experiment 1: Just as a bottom-up interpretation of the results of Experiment 1 led to specific predictions regarding the findings obtained in Experiment 2, a bottom-up interpretation of the results of Experiment 2 also leads to specific predictions regarding the findings obtained in Experiment 1. Namely, if the effects of color salience on focal attention and visual selection were truly dominant and bottom up, these effects should not have been modulated by perceptual load in Experiment 1. At the very least, the magnitude of the distractor interference effect observed in the high-load condition should have been equal across the two experiments when considered under identical trial-to-trial priming conditions (see, e.g., Lamy \& Yashar, 2008, and Pinto et al., 2005). Hence, although a bottom-up account can be applied to the findings obtained in Experiments 1 and 2 when these findings are considered in isolation from one another, such an account cannot be consistently applied to these findings when they are considered together.

\section{GENERAL DISCUSSION}

The scientific study of visual selective attention has enjoyed a long history in experimental psychology, spanning the past century at least (see Driver, 2001; Lavie \& Tsal, 1994; Pashler, 1998; and van der Heijden, 1992, for reviews). Such longevity no doubt speaks to the fundamental role that visual selective attention plays in adaptive functioning, because it allows observers to remain focused on goal-relevant tasks in the presence of distracting stimuli. But how is efficient visual selection accomplished? The present study was conducted to further investigate how visual salience and perceptual load might influence the efficiency of visual selection using a visual search paradigm in which observers attempted to ignore a salient color distractor across high- and low-load conditions. Of critical interest were the findings obtained in the high-load condition, because the effects of color salience and perceptual load were expected to compete in this condition. On one hand, the effects of high perceptual load should have served to eliminate processing of the irrelevant distractor because of the consumption of perceptual resources, whereas on the other hand, the effects of the color singleton should have served to increase processing of the irrelevant distractor because of the attraction of focal attention.

Previous studies using this paradigm have demonstrated that high perceptual load can dominate color salience under some conditions (Gibson \& Bryant, 2008). However, Gibson and Bryant's conclusion was based on a potentially biased measure of distractor interference (I - C as opposed to I - N). Indeed, the results of Experiment 1 reinforced the inadequacy of using $\mathrm{I}-\mathrm{C}$ to estimate the magnitude of distractor interference, although it was also observed that this measure was actually biased against Gibson and Bryant's conclusion, inasmuch as I - C appeared to underestimate the magnitude of distractor interference observed in the low-load (but not in the high- 
load) condition. Thus, the use of I - C did not appear to contaminate Gibson and Bryant's earlier conclusion that perceptual load can dominate color salience, at least under some conditions. Nevertheless, I - N was used exclusively to estimate the magnitude of distractor interference in the present experiments, so that the relative contributions of both color salience and perceptual load could be accurately measured.

In addition, Gibson and Bryant (2008) did not thoroughly investigate whether the relative influence of color salience could be enhanced in certain contexts in which attentional capture by color singletons may be contingent on task knowledge. Accordingly, in the present study, we investigated whether the effects of color salience could be contingent on either or both of two processing sets: feature-processing sets (singleton detection vs. feature search; Bacon \& Egeth, 1994) and attention-allocation sets (wide vs. narrow focus of attention; Belopolsky et al., 2007).

The role of feature-processing sets was examined by investigating whether the magnitude of the distractor interference effect observed in the high-load condition varied as a function of observers' knowledge of the color of the relevant and irrelevant items, whereas the role of attention-allocation sets was examined by investigating whether the magnitude of the distractor interference effect observed in the high-load condition varied as a function of observers' knowledge of the load of the upcoming trial. In both cases, the full-knowledge condition was made possible by blocking the relevant stimulus parameters, whereas the no-knowledge condition was made possible by randomly mixing the relevant stimulus parameters. Note that in Experiment 1, we compared full knowledge with no knowledge of color under conditions of no knowledge of load, whereas in Experiment 2, we compared full knowledge with no knowledge of color under conditions of full knowledge of load.

The results of Experiment 1 suggested that the color distractor's ability to cause interference in the high-load condition was contingent on the attention-allocation set of the observer but not on the feature-processing set. In particular, the present findings were interpreted to suggest that significant distractor interference was observed in the high-load condition only when the observers were set to distribute their attention widely across the display, rather than more narrowly (Belopolsky et al., 2007; Gibson \& Peterson, 2001; Theeuwes, 2004; Theeuwes et al., 2004).

In Experiment 1, the attention-allocation set was manipulated on the basis of the display load shown on trial $n-1$, which then carried over to trial $n$. However, this is not the only way that attention-allocation sets can be manipulated. For instance, Belopolsky et al. (2007) manipulated the width of attention within the context of the additional singleton paradigm (Theeuwes, 1992) by instructing observers to discriminate different aspects of the display: Discriminating the shape of the global search display caused a relatively wide distribution of attention, whereas discriminating the shape of the fixation marker caused a relatively narrow distribution of attention. Con- sistent with their expectations, they found that focal attention was more likely to be captured by an irrelevant color singleton when observers were instructed to discriminate the shape of the global search display than when they were instructed to discriminate the shape of the fixation marker. Note, however, that Belopolsky et al. did not investigate the relative effects of color salience and perceptual load on visual selection in their study, but their method of manipulating the width of attention may prove useful in future studies of this issue.

An important design feature of Experiment 1 was that it allowed for interactions between feature-processing sets and attention-allocation sets, yet no significant interactions were observed. As such, the results obtained in Experiment 1 provide strong evidence that attention-allocation sets can influence the magnitude of attentional capture by color singletons, independent of feature-processing sets (Theeuwes, 2004). In this regard, it should be noted that the inability of color knowledge to modulate the magnitude of attentional capture in Experiment 1 is not due to the ineffectiveness of the knowledge-of-color manipulation. Recall that RTs were significantly slower overall in the noknowledge-of-color condition than in the full-knowledgeof-color condition. This finding in turn suggests that the observers did utilize color knowledge to help parse the search display into relevant and irrelevant items; thus, the observers should have been more vulnerable to the irrelevant singleton in the no-knowledge-of-color condition (singleton detection) than in the full-knowledge-of-color condition (feature search) if feature-processing strategies were capable of influencing attentional capture by color singletons in this paradigm (Bacon \& Egeth, 1994).

Moreover, because the magnitude of the distractor interference effect was reduced equally across the two knowledge-of-color conditions as load increased, the findings obtained in Experiment 1 also provided important new evidence that attentional capture by salient color singletons can be overridden (or at least dampened), even when observers are in singleton detection mode. Thus, singleton detection mode is not sufficient for attentional capture, at least when it is opposed by perceptual load.

Following Theeuwes et al. (2004), the results obtained in Experiment 1 were interpreted to show that attentionallocation sets can influence the magnitude of the distractor interference effect observed in the high-load condition when display load is mixed within blocks. In Experiment 2, we examined the source of the observed modulation more closely by investigating whether the intertrial contingency effects observed in Experiment 1 might reflect the operation of bottom-up priming rather than top-down set by fixing display load within blocks. The change from no knowledge of load in Experiment 1 to full knowledge of load in Experiment 2 produced two distinctly different patterns of findings when the trial-to-trial load sequences were equated (i.e., when low-load displays preceded lowload displays and high-load displays preceded high-load displays). In particular, when trial-to-trial load sequences were equated, perceptual load was found to dominate visual selection when display load was mixed within blocks 
(no knowledge of load), whereas color salience was found to dominate visual selection when display load was fixed within blocks (full knowledge of load).

Because the effect of distractor interference observed in the high-load condition appeared to vary solely as a function of task context in Experiments 1 and 2, these findings were naturally interpreted to be inconsistent with a bottom-up account of visual selection. However, because the results obtained in Experiment 2 deviated from those expected on the basis of the intertrial contingencies observed in Experiment 1, it appears that manipulating knowledge of load had a more profound impact on top-down set than originally expected. In other words, if color salience can dominate perceptual load only when attention is widely distributed, the findings obtained in Experiment 2 can be interpreted to suggest that a widedistribution set may operate by default when load is blocked, but not when load is mixed. Indeed, the effect of distractor interference observed in the high-load condition of Experiment 2 was very similar to the effect of distractor interference that was associated with a wide-distribution set in Experiment 1.

However, attributing the observed changes in selection efficiency to changes in observers' knowledge of load may not be appropriate. For instance, increases in knowledge are typically associated with increases in selection efficiency (see Müller, Geyer, Zehetleitner, \& Krummenacher, 2009 , for a recent example), but the findings obtained in Experiment 2 showed that selection efficiency actually decreased as perceptual load became more predictable in the high-load condition. Thus, fixing load within blocks may have produced other changes in task context besides simply providing advance knowledge of load, which in turn may have induced other search strategies. Evidence in support of this conclusion was provided by the nonsignificant effect of color knowledge in Experiment 2. Unlike those in Experiment 1, the observers in Experiment 2 were able to search equally fast, regardless of whether they knew the color of the target (and nontargets).

Finally, it should be noted that other researchers of attentional capture have also recently reported unexpected effects of task context following blocking versus mixing of specific task parameters. For instance, Lamy and Yashar (2008) investigated the intertrial contingency effects involving trial-to-trial sequences of same or different target shapes reported by Pinto et al. (2005) using the additional singleton paradigm under conditions in which the presence versus absence of the irrelevant color singleton was either blocked or mixed. Consistent with Pinto et al.'s findings, Lamy and Yashar found that trialto-trial changes in target shape led to greater distraction effects relative to trial-to-trial repetitions of target shape under conditions in which advance knowledge of singleton presence was provided. However, these same trialto-trial changes led to the opposite pattern of distraction effects under conditions in which no advance knowledge of singleton presence was provided. Lamy and Yashar interpreted this changing pattern of context effects to be inconsistent with a bottom-up priming account, but they did not explain how knowledge of singleton presence could modulate the observed pattern of intertrial priming. Clearly, future studies will be required to isolate the critical aspects of task context responsible for eliciting such striking changes in attentional capture following the blocking versus mixing of task parameters such as perceptual load and distractor presence.

In conclusion, in the present study, we have extended Gibson and Bryant's (2008) initial investigation of the role of color salience and perceptual load in visual selection. Consistent with those in Gibson and Bryant's study, the present findings also showed that perceptual load can dominate color salience, but only in certain contexts in which display load was mixed and high-load displays preceded other high-load displays. More important, the present findings also showed that perceptual load does not always dominate color salience. Rather, color salience was observed to dominate perceptual load in certain contexts in which display load was mixed and low-load displays preceded high-load displays. In addition, color salience was also observed to dominate perceptual load in contexts in which display load was fixed. Altogether, the present findings demonstrate that the competition between color salience and perceptual load can vary as a function of task context. As such, the present findings appear to be inconsistent with purely bottom-up accounts of color salience and perceptual load, although the precise aspect of task context that controls these contingencies remains to be identified.

\section{AUTHOR NOTE}

The authors thank Gregory J. Davis, Bradley A. Dobrzenski, Daniel Fanuele, and Ann C. Flies for their help with data collection. In addition, the authors also thank Lisa Fournier, Jan Theeuwes, Jeremy Wolfe, and an anonymous reviewer for their comments on this article. Correspondence concerning this article should be addressed to A. T. Biggs or B. S. Gibson, 118 Haggar Hall, Department of Psychology, University of Notre Dame, Notre Dame, IN 46556 (e-mail: abiggs2@nd.edu or bgibson@nd.edu).

\section{REFERENCES}

Bacon, W. F., \& Egeth, H. E. (1994). Overriding stimulus-driven attentional capture. Perception \& Psychophysics, 55, 485-496.

Belopolsky, A. V., Zwaan, L., Theeuwes, J., \& Kramer, A. F. (2007). The size of an attentional window modulates attentional capture by color singletons. Psychonomic Bulletin \& Review, 14, 934-938.

BJork, E. L., \& MurRaY, J. T. (1977). On the nature of input channels in visual processing. Psychological Review, 84, 472-484.

Burnham, B. R. (2007). Displaywide visual features associated with a search display's appearance can mediate attentional capture. Psychonomic Bulletin \& Review, 14, 392-422.

Cosman, J. D., \& Vecera, S. P. (2009). Perceptual load modulates attentional capture by abrupt onsets. Psychonomic Bulletin \& Review, 16, 404-410.

DrIVER, J. (2001). A selective review of selective attention research from the past century. British Journal of Psychology, 92, 53-78.

Eltiti, S., Wallace, D., \& Fox, E. (2005). Selective target processing: Perceptual load or distractor salience? Perception \& Psychophysics, 67, 876-885.

ERIKSEN, C. W., \& ERIKSEN, B. A. (1979). Target redundancy in visual search: Do repetitions of target within the display impair processing? Perception \& Psychophysics, 26, 195-205.

EsTES, W. K. (1972). Interactions of signal and background variables in visual processing. Perception \& Psychophysics, 12, 278-286.

FolK, C. L., Remington, R. W., \& Johnston, J. C. (1992). Involuntary covert orienting is contingent on attentional control settings. Journal 
of Experimental Psychology: Human Perception \& Performance, 18, 1030-1044.

Forster, K. I., \& Forster, J. C. (2003). DMDX: A Windows display program with millisecond accuracy. Behavior Research Methods, Instruments, \& Computers, 35, 116-124.

FourNier, L. R., \& SHORTER, S. (2001). Is evidence for late selection due to automatic or attentional processing of stimulus identities? Perception \& Psychophysics, 63, 991-1003.

Gibson, B. S., \& BRYANT, T. A. (2008). The identity intrusion effect: Attentional capture or perceptual load? Visual Cognition, 16, 182-199.

Gibson, B. S., \& Kelsey, E. M. (1998). Stimulus-driven attentional capture is contingent on attentional set for displaywide visual features. Journal of Experimental Psychology: Human Perception \& Performance, 24, 699-706.

Gibson, B. S., \& Peterson, M. A. (2001). Inattentional blindness and attentional capture: Evidence for attention-based theories of visual salience. In C. L. Folk \& B. S. Gibson (Eds.), Attraction, distraction, and action: Multiple perspectives on attentional capture (pp. 51-76). New York: Elsevier.

Lamy, D., \& Egeth, H. E. (2003). Attentional capture in singletondetection and feature-search modes. Journal of Experimental Psychology: Human Perception \& Performance, 29, 1003-1020.

LAMY, D., \& YASHAR, A. (2008). Intertrial target-feature changes do not lead to more distraction by singletons: Target uncertainty does. Vision Research, 48, 1274-1279.

LaVIE, N. (1995). Perceptual load as a necessary condition for selective attention. Journal of Experimental Psychology: Human Perception \& Performance, 21, 451-468.

Lavie, N. (2005). Distracted and confused? Selective attention under load. Trends in Cognitive Sciences, 9, 75-82.

LaVIE, N., \& Cox, S. (1997). On the efficiency of visual selection attention: Efficient visual search leads to inefficient distractor rejection. Psychological Science, 8, 395-398.

Lavie, N., \& Tsal, Y. (1994). Perceptual load as a major determinant of the locus of selection in visual attention. Perception \& Psychophysics, 56, 183-197.

Loftus, G. R., \& Masson, M. E. J. (1994). Using confidence intervals in within-subjects designs. Psychonomic Bulletin \& Review, 1, 476490.

Miller, J. (1982). Divided attention: Evidence for coactivation with redundant signals. Cognitive Psychology, 14, 247-279.

Müller, H. J., Geyer, T., Zehetleitner, M., \& Krummenacher, J. (2009). Attentional capture by salient color singleton distractors is modulated by top-down dimensional set. Journal of Experimental Psychology: Human Perception \& Performance, 35, 1-16.

PASHLER, H. (1998). The psychology of attention. Cambridge, MA: MIT Press.

Pinto, Y., Olivers, C. N. L., \& Theeuwes, J. (2005). Target uncer- tainty does not lead to more distraction by singletons: Intertrial priming does. Perception \& Psychophysics, 67, 1354-1361.

Santee, J. L., \& Egeth, H. E. (1982). Do reaction time and accuracy measure the same aspects of letter recognition? Journal of Experimental Psychology: Human Perception \& Performance, 8, 489-501.

Theeuwes, J. (1991). Cross-dimensional perceptual selectivity. Perception \& Psychophysics, 50, 184-193.

Theeuwes, J. (1992). Perceptual selectivity for color and form. Perception \& Psychophysics, 51, 599-606.

TheEuwes, J. (1994). Stimulus-driven capture and attentional set: Selective search for color and visual abrupt onsets. Journal of Experimental Psychology: Human Perception \& Performance, 20, 799-806.

Theeuwes, J. (2004). Top-down search strategies cannot override attentional capture. Psychonomic Bulletin \& Review, 11, 65-70.

Theeuwes, J., \& Burger, R. (1998). Attentional control during visual search: The effect of irrelevant singletons. Journal of Experimental Psychology: Human Perception \& Performance, 24, 1342-1353.

Theeuwes, J., Kramer, A. F., \& Belopolsky, A. V. (2004). Attentional set interacts with perceptual load in visual search. Psychonomic Bulletin \& Review, 11, 697-702.

Torralbo, A., \& BeCK, D. M. (2008). Perceptual-load-induced selection as a result of local competitive interactions in visual cortex. Psychological Science, 19, 1045-1050.

VAN DER HeIJDEN, A. H. C. (1992). Selective attention in vision. New York: Routledge.

\section{NOTE}

1. The intertrial contingencies observed in Experiment 1 also help rule out an alternative explanation of the overall perceptual load $\times$ distractor compatibility interaction in which the magnitude of the distractor interference effect is determined by the dynamics of serial search, as opposed to the consumption of perceptual resources. According to this serial search account, the likelihood that the salient distractor will be processed is determined by the likelihood that the distractor will be randomly sampled during the course of serial search. According to this account, the effect of distractor interference may be larger in the low-load condition than in the high-load condition simply because the distractor is more likely to be randomly sampled when there are 4 items in the display than when there are 12 items in the display. However, the serial search account cannot explain why the effect of distractor interference observed in the high-load condition of Experiment 1 depended on the number of items appearing in the preceding display, whereas the effect of distractor interference observed in the low-load condition of Experiment 1 did not depend on this factor.

(Manuscript received August 2, 2008; revision accepted for publication August 2, 2009.) 